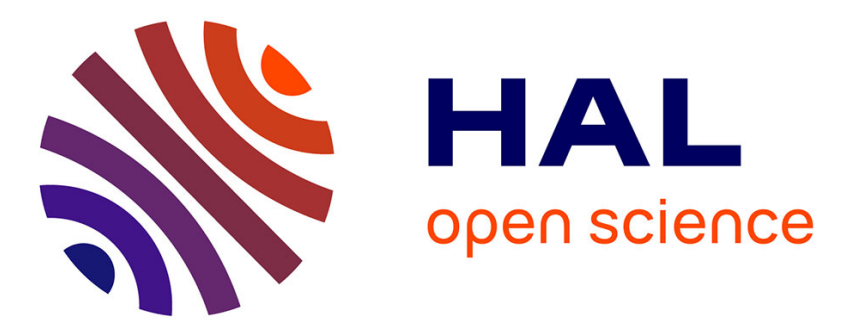

\title{
Prediction-Based Control of Linear Systems by Compensating Input-Dependent Input Delay of Integral-Type
}

\author{
Delphine Bresch-Pietri, Nicolas Petit
}

\section{- To cite this version:}

Delphine Bresch-Pietri, Nicolas Petit. Prediction-Based Control of Linear Systems by Compensating Input-Dependent Input Delay of Integral-Type. I. Karafyllis, M. Malisoff, F. Mazenc and P. Pepe. Recent Results on Nonlinear Delay Control Systems, , 2015, 10.1007/978-3-319-18072-4_4 . hal01227313

\section{HAL Id: hal-01227313 \\ https://hal.science/hal-01227313}

Submitted on 10 Nov 2015

HAL is a multi-disciplinary open access archive for the deposit and dissemination of scientific research documents, whether they are published or not. The documents may come from teaching and research institutions in France or abroad, or from public or private research centers.
L'archive ouverte pluridisciplinaire HAL, est destinée au dépôt et à la diffusion de documents scientifiques de niveau recherche, publiés ou non, émanant des établissements d'enseignement et de recherche français ou étrangers, des laboratoires publics ou privés. 


\title{
Prediction-Based Control of Linear Systems by Compensating Input-Dependent Input Delay of Integral-Type
}

\author{
Delphine Bresch-Pietri and Nicolas Petit
}

\begin{abstract}
This study addresses the problem of delay compensation via a predictorbased output feedback for a class of linear systems subject to input delay which itself depends on the input. The equation defining the delay is implicit and involves past values of the input through an integral relation, the kernel of which is a polynomial function of the input. This modeling represents systems where transport phenomena take place at the inlet of a system involving a nonlinearity, which frequently occurs in the processing industry. The conditions of asymptotic stabilization require the magnitude of the feedback gain to comply with the initial conditions. Arguments for the proof of this novel result include general Halanay inequalities for delay differential equations and take advantage of recent advances in backstepping techniques for uncertain or varying delay systems.
\end{abstract}

\section{Introduction}

Numerous control systems involve a physical dead-time which proves to be troublesome in the design and tuning of feedback control laws. The dead-time results from the fact that sensors and actuators are rarely co-located, for example in processes that involve the transport of materials, such as mixing processes for liquid or gaseous fluids, chemical reactors [12], automotive engine and exhaust lines [9], heat collector plants [24], networks for blending liquids and solids [8], and batch processes [22]. In all of these, and also in the crushing mill system described by [23], the lag directly depends on the control variable and is inherently input-dependent.

Delphine Bresch-Pietri,

CNRS at GIPSA-lab, Control Department, 11 rue des Mathématiques, 38000 Grenoble FRANCE

e-mail: delphine.bresch-pietriegipsa-lab.fr

Nicolas Petit,

Centre Automatique et Systèmes at MINES ParisTech, 60 boulevard Saint-Michel, 75006 Paris

FRANCE e-mail: nicolas.petit@mines-paristech.fr 
It seems that the stabilization of such processes with input-dependent time-delay in the input $D(u)$ or $D\left(u_{t}\right)$, where $u_{t}$ denotes past values over a finite horizon, has seldom been theoretically studied. Rather, a widely considered approach is to recast the delay dependence on the control, e.g., by modeling this dependence as $D(u) \approx$ $D(t)$ (or even by a constant average value $D$ ) and expecting the controller to deal with a certain level of unstructured model variability. The advantage of such an approach is that it enables the use of a wide range of technical tools previously developed to deal with disturbances. However, recasting this input-dependency into the more general class of input-disturbance reduces performance potential. For this reason we propose to explicitly take this dependency into account and to employ a prediction-based controller, in an effort of improving transient performances.

Predictor-based control strategies $[1,17,25]$, which are state-of-the-art for systems with constant input time-delays (as studied in $[3,10,14,18,19]$ or [23] and the references therein) are much more complex to apply for time-varying delays. As shown in [20] or, more recently, in [16], to compensate a time-varying input delay, the prediction has to be calculated over a time window whose length matches the value of the future delay. In other words, future variations in the delay must be predicted. This can be done, for example, when one has access to a given delay model as in [27] or when the delay is state-dependent, by carefully predicting the future system state, as proposed in [2].

Yet, when the delay depends on the input, things become very involved. Determining the required prediction horizon is then an implicit issue, which in practice may not be resolvable or even well-posed. This implicit nature is caused by the reciprocal interactions between the (current and past) control values and the delay, yielding a closed-loop dependency.

In order to design such a prediction-based control law, we advocate a two-step method of disrupting the implicit loop, as proposed in our recent works on the topic $[4,6]$ and establish sufficient conditions for asymptotic stabilization.

In a first step, we considered the input-dependency as a particular form of timevariation. It enables us to then use a robust compensation result for linear systems with time-varying input delay, using the backstepping tools proposed in [15] for the analysis of input-delay system stability. Technically, this guarantees stabilization, provided the delay variations are sufficiently small. Second, a sufficient bound on the delay variations was obtained by relating them to the control tracking error, which is analyzed using the asymptotic convergence of delay differential equations (DDE) (Halanay-type inequalities [11]).

The result holds for potentially unstable linear systems of any arbitrary order $n \in$ $\mathbb{N}$. Assuming that the kernel defining the integral is a positive polynomial function of the input, our previous result was extended to such a framework. This is the main novelty of this study. As shown, this delay model encompasses a large class of transport processes. For this class of systems, robust compensation is shown to be achievable, provided that the initial conditions are sufficiently close to equilibrium and that the feedback gain is chosen accordingly.

This chapter is organized as follows. In Section 2, the problem is illustrated by a rocket engine system as an motivating example. A prediction-based controller is 
designed in Section 3. Sufficient conditions for stabilization are derived through a proof of convergence, calling on Halanay-like inequalities arguments in Section 4.

Notation and Definitions. In the following, |.| stands for the usual Euclidean norm, and $\mathscr{C}^{0}\left(S_{1}, S_{2}\right)$ denotes the set of all continuous functions on a set $S_{1}$ with values into a set $S_{2}$. For a given symmetric matrix, $\underline{\lambda}($.$) and \bar{\lambda}($.$) stand respectively$ for minimum and maximum eigenvalues.

Classically, $x_{t}$ refers to the function $x_{t}:[-\bar{D}, 0] \rightarrow x(t+s)$ defined by $x_{t}(s)=$ $x(t+s)$ for a given function $x$ and $\bar{D}>0$ and $\max \left|x_{t}\right|=\max \left\{\left|x_{t}(s)\right|: s \in[-\bar{D}, 0]\right\}$.

For any bounded function $k$ defined on $[-\bar{D}, 0]$ and any polynomial function $\pi$, we write

$$
\pi\left(x_{t}\right)=\left(x\left(t_{1}\right), \ldots, x\left(t_{n-2}\right), \int_{t_{n-1}}^{t_{n}} k(t-s) x(s) d s\right)
$$

for $\left(t_{1}, \ldots, t_{n}\right) \in[t-\bar{D}, t]^{n}$. We also refer to $\pi\left(x_{t}\right)$ as a polynomial function in $x_{t}$.

A polynomial function $\pi$ in the variables $\left(x_{1}, \ldots, x_{n}, x_{n+1}\right)$ is said to be at least quadratic in $x_{1}, \ldots, x_{n}$ if, for any given $x_{n+1}$, the corresponding polynomial function $\pi_{x_{n+1}}$ defined as

$$
\pi_{x_{n+1}}\left(x_{1}, \ldots, x_{n}\right)=\pi\left(x_{1}, \ldots, x_{n}, x_{n+1}\right)
$$

has no terms of order 0 or 1, e.g., $\pi=x_{1}^{2}+x_{1} x_{2} x_{3}$ and $\pi=x_{2} x_{1}+x_{3} x_{1}^{2}$ are both at least quadratic in $\left(x_{1}, x_{2}\right)$ while $\pi=x_{3}+x_{3} x_{2}^{2}$ is not.

\section{Problem Statement}

Consider the following potentially unstable linear time-invariant plant sketched in Fig. 1:

$$
x^{(n)}(t)+a_{n-1} x^{(n-1)}(t)+\ldots+a_{1} \dot{x}(t)+a_{0} x(t)=b_{0} u(t-D(t))
$$

driven by a delayed input, where the varying delay $D(t)$ is implicitly defined in terms of the input history by

$$
\int_{t-D(t)}^{t} \varphi(u(s)) d s=1,
$$

in which $\varphi: \mathbb{R} \mapsto[\varphi, \infty)$ is a polynomial function with positive values (i.e., $\varphi>0$ ). Physically, the integral equation (3) corresponds to a plug-flow assumption [21] in a transport phenomenon and appears in a large class of applications. The variable $\varphi$ is a (normalized) flow rate, which depends polynomially on the system input. Since $\varphi \geq \underline{\varphi}>0$, this transport delay is well defined ${ }^{1}$ and is upper-bounded, as follows:

1 The delay is positive and, besides, its derivative can be expressed as $\dot{D}(t)=1-\varphi(u(t)) /(\varphi(u(t-D)))<1$ which guarantees strict causality. 


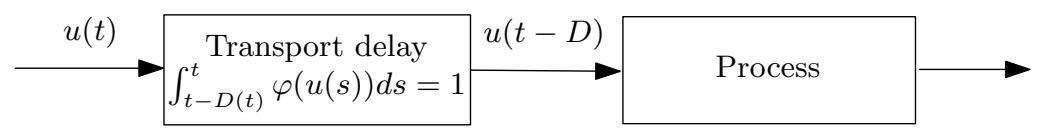

Fig. 1 The addressed problem, where the input is delayed by a transport delay which is inputvarying.

$$
D(t) \leq \bar{D}=1 / \underline{\varphi} \text { for all } t \geq 0
$$

In particular, as

$$
D \mapsto \int_{t-D}^{t} \varphi(u(s)) d s
$$

is strictly increasing, it is invertible and the delay can be calculated and so is assumed to be known in the following.

Motivating Example Consider a liquid non-hypergolic propellant rocket such as the one pictured on Fig. 2. The fuel and oxidizer are stored in separate tanks, blended and then fed through a system of pipes, valves, and turbopumps to a com-

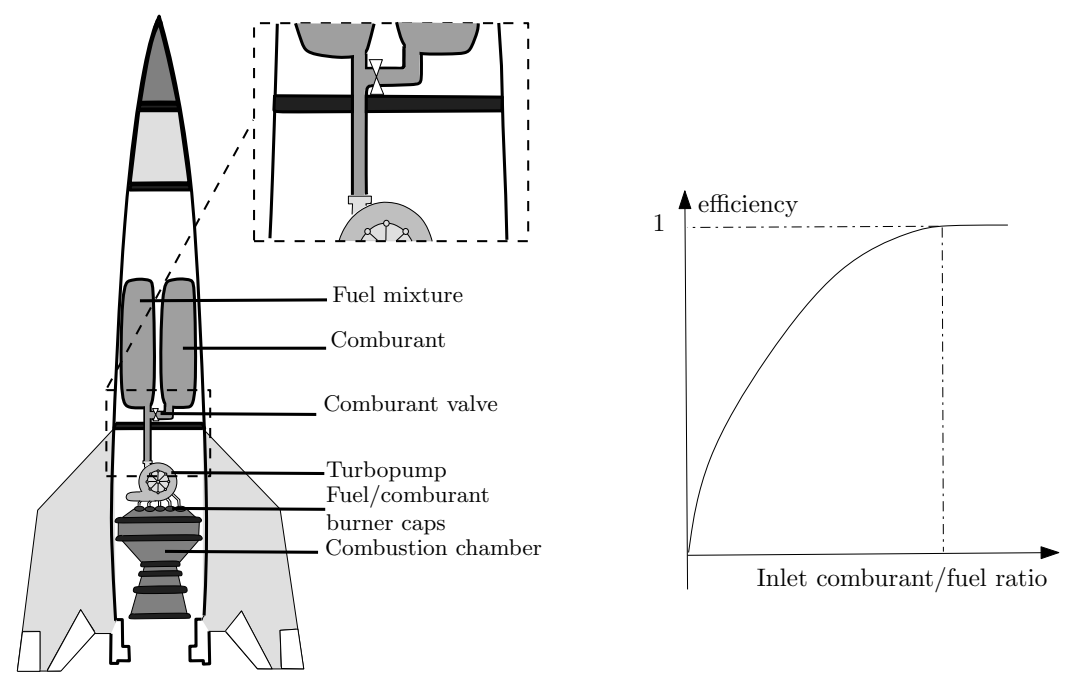

Fig. 2 Left: Schematic view of a low cost designed (single choke) bipropellant rocket engine. Zoom in on the transport phenomenon in the top right corner. Right: Schematic view of the evolution of the combustion efficiency with respect to the comburant/fuel ratio entering the chamber.

bustion chamber, where they are burned to generate thrust. As the mixture is nonhypergolic, combustion is started by an ignition system in the chamber. Compared to more classical setups (with dual pumps and pipes convecting the tanks directly 
to the combustion chamber), this architecture has been chosen for cost reasons, as it uses only one pipe, one turbopump and one choke.

After being premixed downstream of the tanks, the mixture flows to the combustion chamber, which generates a transport delay satisfying

$$
\int_{t-D(t)}^{t} \frac{Q(s)}{V_{P}} d s=1,
$$

in which $V_{P}$ is the pipe holdup and $Q(s)=Q_{1}+Q_{2}(s)$ is the total volume flow rate after mixing, i.e., the sum of the (constant) outlet fuel flow rate and the comburant flow rate. Following Newton's law, the (longitudinal) dynamics of the rocket can therefore be written as

$$
\ddot{x}(t)=\eta(t) F_{0},
$$

in which $F_{0}$ is the optimal thrust, corresponding to a stoichiometric mixture, and $\eta$ is the combustion efficiency. The latter is significantly nonlinear at high flow-rate ratios, which vanishes for low ratios [26]. Hence, it can reasonably be modeled as $\eta(t)=\eta_{0} \sqrt{Q_{2}(t-D(t))}$ for example (without loss of generality), with the oxygen volume flow rate $Q_{2}(t-D(t)$ entering the combustion chamber. Therefore, accounting for (6) and setting

$$
u(t)=\eta_{0} F_{0} \sqrt{Q_{2}(t)}
$$

one obtains

$$
\left\{\begin{array}{l}
\ddot{x}(t)=u(t-D(t)) \\
\int_{t-D(t)}^{t} \frac{1}{V_{P}}\left(Q_{1}+\frac{u(s)^{2}}{\eta_{0}^{2} F_{0}^{2}}\right) d s=1
\end{array}\right.
$$

which is indeed in the form (2)-(3) with

$$
\varphi(u(s))=\frac{1}{V_{P}}\left(Q_{1}+\frac{u(s)^{2}}{\eta_{0}^{2} F_{0}^{2}}\right)
$$

being a second-order polynomial function with strictly positive values. More complex polynomial expressions can be considered to account for more detailed fit of experimental data and thrust maps.

Control Design Objective The control task is to stabilize the plant at any equilibrium point $x^{r}$ such that $x^{r}=b_{0} / a_{0} u^{r}$, where $u^{r}$ is the corresponding input. For this purpose, a predictor-based feedback law will be employed here.

With this aim in view, we first formulate a state-space representation of this system as

$$
\left\{\begin{array}{l}
\dot{X}(t)=A X(t)+B u(t-D(t)) \\
\int_{t-D(t)}^{t} \varphi(u(s)) d s=1
\end{array}\right.
$$


where

$$
A=\left(\begin{array}{cccc}
0 & 1 & & 0 \\
\vdots & & \ddots & \\
0 & 0 & & 1 \\
-a_{0} & -a_{1} & \ldots & -a_{n-1}
\end{array}\right) \text { and } B=\left(\begin{array}{c}
0 \\
\vdots \\
0 \\
b_{0}
\end{array}\right)
$$

For the sake of clarity, we assume in the following that the system state $X$ is fully measured. Extending this to output feedback for observable systems is straightforward, following the methodology of [7]. To construct a prediction-based control law, we use the following theorem, a proof of which is given in [4] and [5].

Theorem 1 (Step 1: Preliminary result). Consider the closed-loop single input system

$$
\begin{aligned}
\dot{X}(t) & =A X(t)+B u(t-D(t)) \\
u(t) & =K\left[e^{A D(t)} X(t)+\int_{t-D(t)}^{t} e^{A(t-s)} B u(s) d s\right],
\end{aligned}
$$

where $X \in \mathbb{R}^{n}, u \in \mathbb{R}, K$ is chosen such that $A+B K$ is Hurwitz, and $D: \mathbb{R}_{+} \rightarrow[0, \bar{D}]$ is a time-differentiable function. Define

$$
\Upsilon_{0}(t)=|X(t)|^{2}+\int_{t-D(t)}^{t} u(s)^{2} d s+D(t)^{2} \int_{t-D(t)}^{t} \dot{u}(s)^{2} d s .
$$

There exists $\Delta^{*}(K) \in(0,1)$ such that, if

$$
|\dot{D}(t)|<\Delta^{*}(K), \quad t \geq 0,
$$

then the plant (10a) exponentially converges to the origin, in the sense that there exist $R>0$ and $\rho>0$ such that $\Upsilon_{0}(t) \leq R \Upsilon_{0}(0) e^{-\rho t}$ for all $t \geq 0$.

The prediction controller (10b) is a natural extension from the case of constant delay. It forecasts values of the state over a time window of varying length $D(t)$. Of course, exact compensation of the delay is not achieved with this controller. To do so, one would need to consider a time window whose length exactly matched the value of the future delay, as done in [20] and [16]. In detail, defining $\eta(t)=t-D(t)$ and assuming that its inverse exists ${ }^{2}$, exact delay-compensation is obtained form the feedback law $U(t)=K X\left(\eta^{-1}(t)\right)$. Yet, implementing this relation requires the future variation of the delay to be predicted via $\eta^{-1}(t)$, which is not achievable in practice for an input-varying delay.

Equation (11) can be interpreted as a condition for achieving robust delay compensation $^{3}$. The essence of this condition is that, if the delay were to vary sufficiently

\footnotetext{
2 This is the case if $\dot{D}<1$.

${ }^{3}$ Interestingly, a similar condition is often stated in Linear Matrix Inequality approaches, such as [28] for example, where the delay is also assumed to be time-differentiable.
} 
slowly, its current value $D(t)$ used for prediction would remain sufficiently close to its future values, and the corresponding prediction would be accurate enough to guarantee the stabilization of the plant through the feedback loop.

We now focus on an alternative sufficient condition guaranteeing (11) when the delay varies in accordance with the integral relation (3). For practical control design, this new condition involves the control gain and the initial conditions.

\section{Control Design}

In this section, we now establish a sufficient condition guaranteeing (11) when the delay varies in accordance with the integral relation (3). This new condition is relevant to the initial conditions (and thus, to the choice of control gain).

Theorem 2. Consider the closed-loop system

$$
\begin{aligned}
& \dot{X}(t)=A X(t)+B u(t-D(t)) \\
& \int_{t-D(t)}^{t} \varphi((u(s)) d s=1 \\
& u(t)=u^{r}+K\left[e^{A D(t)} X(t)+\int_{t-D(t)}^{t} e^{A(t-s)} B u(s) d s-X^{r}\right] .
\end{aligned}
$$

where $\varphi: \mathbb{R} \mapsto[\varphi, \infty)$ is a polynomial function, with $\varphi>0$ constant, $A$ and $B$ are defined in (9), $K$ is such that $A+B K$ is Hurwitz, $u$ is scalar, $X^{r}$ is the state equilibrium corresponding to the original equilibrium $x^{r}$ of the plant (2) and $u^{r}$ is the corresponding (constant) reference control. Consider the functionals

$$
\begin{aligned}
& \Theta(t)=\left|X(t)-X^{r}\right|+\max _{s \in[t-\bar{D}, t]}\left|\left[u(s)-u^{r} \dot{u}(s) \ldots u^{(n-1)}(s)\right]^{T}\right| \\
& \Upsilon(t)=\left|X(t)-X^{r}\right|^{2}+\int_{t-D(t)}^{t}\left(u(s)-u^{r}\right)^{2} d s+D(t)^{2} \int_{t-D(t)}^{t} \dot{u}(s)^{2} d s .
\end{aligned}
$$

Provided that $u_{0} \in \mathscr{C}^{n}([-\bar{D}, 0], \mathbb{R})$, there exists $\theta: \mathbb{R}^{n} \mapsto \mathbb{R}_{+}^{\star}$ such that, if $\Theta(0)<$ $\theta(K)$, then condition (11) is fulfilled and the plant exponentially converges to $X^{r}$ in the sense that there exist $R>0$ and $\rho>0$ such that $\Upsilon(t) \leq R \Upsilon(0) e^{-\rho t}$ for all $t \geq 0$.

This result has a relatively direct interpretation: the previously presented Theorem 1 requires the delay to vary sufficiently slowly, while on the other hand, the delay variations implicitly depend on the control input through the integral equation (3), with variations whose rapid swings are scaled by the gain $K$. Therefore, it would seem to be a natural requirement to restrict input variations by choosing initial conditions sufficiently near the desired equilibrium and which comply with the magnitude of the feedback gain.

The behavior of $\theta$ with respect to $K$ must be investigated in future work. From the expression (31), this would involve a study of the solution of the Lyapunov 
equation and the behavior of its eigenvalues with respect to $K$. One would expect that $\theta(K) \rightarrow 0$ while $K \rightarrow-\infty$ (since $r(K)$ is expected in this case to tend to $\infty$ ). For this reason, for given initial conditions, the magnitude of the feedback gain should be chosen accordingly. For relatively large initial conditions, this would imply the use of a small feedback gain; therefore, this result can be interpreted as a small-gain condition. We now detail the proof of Theorem 2 .

\section{Proof of Theorem - Halanay-like Inequalities}

We prove Theorem 2. Taking a time-derivative of (13) and defining the error variable $\varepsilon=u-u^{r}$, one gets

$$
\dot{D}(t)=\frac{\varphi(u(t-D(t)))-\varphi(u(t))}{\varphi(u(t-D(t)))}=\frac{\varphi\left(\varepsilon(t-D(t))+u^{r}\right)-\varphi\left(\varepsilon(t)+u^{r}\right)}{\varphi(u(t-D(t)))} .
$$

As $\varphi$ is a polynomial function, it is locally Lipschitz with a constant $M(\kappa)$ on the interval $[-\kappa, \kappa]$ for any positive scalar $\kappa$. Then, since $\varphi \geq \underline{\varphi}$, we obtain

$$
\dot{D}(t) \leq \frac{M(\kappa)\left|\varepsilon(t-D(t))+u^{r}-\left(\varepsilon(t)+u^{r}\right)\right|}{\underline{\varphi}}=\frac{M(\kappa)|\varepsilon(t-D(t))-\varepsilon(t)|}{\underline{\varphi}} \leq \frac{2 M(\kappa) \max \left|\mathcal{E}_{t}\right|}{\underline{\underline{\varphi}}},
$$

if $\max \left|\varepsilon_{t}\right| \leq \kappa$. Hence, for any positive parameter $\kappa>0$, condition (11) is satisfied if

$$
\forall t \geq 0, \quad \max \left|\varepsilon_{t}\right|<\min \left\{\frac{\varphi \Delta^{*}(K)}{2 M(\kappa)}, \kappa\right\} .
$$

This is the condition we now focus on. It yields the analysis of the dynamics of the variable $\varepsilon$. Before beginning this analysis in detail, we recall some well-known stability results for DDE and their extensions.

\subsection{Halanay Inequality for Delay Equations of Order $n \geq 1$}

We first recall the following result ( [11], [13]) ${ }^{4}$.

Lemma 1. (Halanay inequality) Consider a continuous positive- and real-valued function $x$ such that, for some $t_{0} \in \mathbb{R}$,

$$
\dot{x}(t) \leq-a x(t)+b \max x_{t}, \quad t \geq t_{0}
$$

with $a \geq b \geq 0$. Then, there exists $\gamma \geq 0$ such that

$$
\forall t \geq t_{0}, \quad x(t) \leq \max x_{t_{0}} e^{-\gamma\left(t-t_{0}\right)}
$$

\footnotetext{
${ }^{4}$ More precisely, in [11], this result is stated for $a>b>0$.
} 
Straightforward extensions of this lemma are stated below.

Corollary 1. Consider a positive-valued continuous function such that

$$
\left\{\begin{aligned}
\dot{x}(t) & \leq-a x(t)+b h\left(t, x_{t}\right), \quad t \geq t_{0} \\
x_{t_{0}} & =\psi \in \mathscr{C}^{0}\left([-\bar{D}, 0], \mathbb{R}_{+}\right)
\end{aligned}\right.
$$

where $h$ is a continuous functional satisfying the sup-norm relation

$$
h\left(t, x_{t}\right) \leq \max \left|x_{t}\right|, \text { for } \max \left|x_{t}\right|<\omega,
$$

for a given $\omega>0$. If the initial condition is such that $\max \psi<\omega$ and if $a \geq b \geq 0$, then there exists $\gamma \geq 0$ (where $\gamma=0$ if $a=b$ and $\gamma>0$ otherwise) such that

$$
\forall t \geq t_{0}, \quad x(t) \leq \max x_{t_{0}} e^{-\gamma\left(t-t_{0}\right)} .
$$

Proof. Consider $\psi$ such that $x$ is a non-trivial continuous solution ${ }^{5}$ of (18) which, using (19), does satisfy the inequality

$$
\dot{x}(t) \leq-a x(t)+b \max x_{t} \quad \text { if } \max x_{t}<\omega .
$$

Following the seminal proof of [11], define $y(t)=k e^{-\gamma\left(t-t_{0}\right)}$, with $k>0$ and $\gamma$ chosen such that $y$ satisfies the corresponding differential equation ${ }^{6}$

$$
\dot{y}(t)=-a y(t)+b \max y_{t}, \quad t \geq t_{0} \quad \text { and } \quad y_{t_{0}}=k .
$$

Now, we define the difference $z=y-x$, which is a continuous function, and we are interested in its sign change. We choose $k \in\left(\max x_{t_{0}}, \omega\right)$, which is a not empty set as $\max x_{t_{0}}=\max \psi<\omega$. This guarantees that $z(t)>0$ for $t \in\left[t_{0}-\bar{D}, t_{0}\right]$. The function $z$ being continuous, we define

$$
t_{1}=\inf \left\{t>t_{0} \mid z(t)=0\right\} \in \mathbb{R} \cup\{\infty\} .
$$

Assume that $t_{1}<\infty$. From the definition of $t_{1}, z(t)>0$ for $t \in\left[t_{0}, t_{1}\right]$ and, from the analytical expression of $y$ and as both $x$ and $y$ are continuous, $x(t)<y(t)<k<\omega$ for $t \in\left[t_{0}, t_{1}\right)$. Therefore, the following inequality holds

$$
\forall t \leq t_{1}, \quad \dot{z}(t) \geq-a z(t)+b\left(\max y_{t}-\max x_{t}\right) .
$$

Then, $\dot{z}\left(t_{1}\right) \geq \max y_{t_{1}}-\max x_{t_{1}}>0$, by definition of $t_{1}$. Yet, one has

$$
\dot{z}\left(t_{1}\right)=\lim _{t \rightarrow t_{1}^{-}} \frac{z(t)-z\left(t_{1}\right)}{t-t_{1}}=\lim _{t \rightarrow t_{1}^{-}} \frac{z(t)}{t-t_{1}} \leq 0 \text { as } z(t) \geq 0 \text { on }\left[t_{0}, t_{1}\right] .
$$

\footnotetext{
${ }^{5}$ The case when $x$ is identically 0 is trivial. The continuity (and even more) is obtained by assuming $\psi$ is smooth enough.

${ }^{6} \gamma \geq 0$ is the unique solution on $[0, \infty[$ of $a-\gamma=b \exp (\gamma \bar{D})$.
} 
We finally conclude that $t_{1}=\infty$. Then, for $t \geq t_{0}$ and all $z(t)>0$ and, for any $\varepsilon=k-\max x_{t_{0}}>0$, we get

$$
\forall t \geq t_{0} \quad x(t)<\left(\max x_{t_{0}}+\varepsilon\right) e^{-\gamma\left(t-t_{0}\right)},
$$

which gives the result.

Lemma 2. [stability of $a n^{\text {th }}$ order DDE] Let $x$ be a solution of the $n^{\text {th }}$ order DDE

$$
\left\{\begin{array}{l}
x^{(n)}(t)+\alpha_{n-1} x^{(n-1)}(t)+\ldots+\alpha_{0} x(t)=c \ell\left(t, x_{t}, \ldots x_{t}^{(n-1)}\right), t \geq t_{0} \\
X_{t_{0}}=\psi \in \mathscr{C}^{0}([-\bar{D}, 0], \mathbb{R}) \quad \text { with } \max |\psi|<\omega,
\end{array}\right.
$$

where the left-hand side of the differential equation defines a polynomial whose roots have only strictly negative real parts, $c>0, \omega>0$ and $\ell$ is a continuous functional. Then, there exist $c_{*}>0$ and $r_{*}>0\left(r_{*}=1\right.$ and $c_{*}=\alpha_{0}$ if $\left.n=1\right)$ such that, if

- $c \leq c_{*}$;

- $\ell$ satisfies the following sup norm relation, with $X=\left[\begin{array}{ll}x & \dot{x} \ldots x^{(n-1)}\end{array}\right]^{T}$,

$$
\left|\ell\left(t, x_{t}, \ldots, x_{t}^{(n-1)}\right)\right| \leq \max \left|X_{t}\right|, \text { for } t \geq t_{0}, \max \left|X_{t}\right|<r \omega \text { with } r \geq r_{*},
$$

then, there exists $\gamma \geq 0$ such that $|X(t)| \leq r_{*} \max \left|X_{t_{0}}\right| e^{-\gamma\left(t-t_{0}\right)}$ holds for all $t \geq 0$.

Proof. Using the scalar result of Corollary 1, define the scalar positive valued function $m(t)=X(t)^{T} P X(t)$ where $P$ is, as defined in the statement of the Lemma, the symmetric positive definite matrix solution of the Lyapunov equation $A_{0}^{T} P+P A_{0}=$ $-Q$, for some given symmetric positive definite matrix $Q$ and

$$
A_{0}=\left(\begin{array}{cccc}
0 & 1 & & \\
\vdots & \ddots & \\
0 & & & 1 \\
-\alpha_{0} & -\alpha_{1} & \ldots & -\alpha_{n-1}
\end{array}\right)
$$

is the companion matrix. Taking a time-derivative of $m$, we obtain

$$
\dot{m}(t)=-X(t)^{T} Q X(t)+2 X(t)^{T} P\left(\begin{array}{c}
0 \\
\vdots \\
0 \\
c \ell\left(t, x_{t}, \ldots, x_{t}^{(n-1)}\right)
\end{array}\right)
$$

Therefore, defining $a \stackrel{\Delta}{=} \frac{\lambda(Q)}{\bar{\lambda}(P)}$ and $b \stackrel{\Delta}{=} 2 c \frac{\bar{\lambda}(P)}{\underline{\lambda}(P)}$, one obtains

$$
\dot{m}(t) \leq-a m(t)+b h(t), \text { where }
$$




$$
\begin{aligned}
h(t) & =\frac{\lambda(P)}{\bar{\lambda}(P)} X(t)^{T} P\left(\begin{array}{c}
0 \\
\vdots \\
0 \\
\ell\left(t, x_{t}, \ldots, x_{t}^{(n-1)}\right)
\end{array}\right) \\
& \leq \underline{\lambda}(P)|X(t)|\left|\ell\left(t, x_{t}, \ldots, x_{t}^{(n-1)}\right)\right| \leq \sqrt{\underline{\lambda}(P)} \sqrt{m(t)}\left|\ell\left(t, x_{t}, \ldots, x_{t}^{(n-1)}\right)\right|,
\end{aligned}
$$

which satisfies the following for $\max \left|X_{t}\right|<r \omega$ (and therefore, in particular, for $\left.\max m_{t}<r^{2} \omega^{2} \underline{\lambda}(P)\right):|h(t)| \leq \sqrt{m(t)} \max \sqrt{m_{t}} \leq \max m_{t}$. Define the condition

$$
\max m_{t_{0}}<r^{2} \omega^{2} \underline{\lambda}(P)
$$

If (24) holds then we conclude by applying Corollary 1 to (22), that, if $a>b$, then there exists $\gamma>0$ such that

$$
\forall t \geq t_{0}, \quad m(t) \leq \max m_{t_{0}} e^{-2 \gamma\left(t-t_{0}\right)} .
$$

A sufficient condition for $\max m_{t_{0}}<r^{2} \omega^{2} \underline{\lambda}(P)$ is that

$$
\max \left|X_{t_{0}}\right|^{2}<r^{2} \omega^{2} \underline{\lambda}(P) / \bar{\lambda}(P) .
$$

By assumption $\left|\max X_{t_{0}}\right|<\omega$. Hence, to guarantee that (24) holds, we simply have to pick

$$
r \geq r_{*} \triangleq \sqrt{\bar{\lambda}(P) / \underline{\lambda}(P)}
$$

Finally, the condition $a>b$ can be reformulated as

$$
c<\frac{\lambda(P) \underline{\lambda}(Q)}{2 \bar{\lambda}(P)^{2}} \triangleq c_{*}
$$

and one obtains

$$
\forall t \geq t_{0}, \quad|X(t)| \leq \sqrt{\frac{\bar{\lambda}(P)}{\underline{\lambda}(P)}} \max \left|X_{t_{0}}\right| e^{-\gamma\left(t-t_{0}\right)}
$$

which concludes the proof.

\subsection{Application to Dynamical Equation Defined through the Predictor-Based Control Law}

We now focus on the DDE governing $\varepsilon$, which is given in the following lemma, the proof of which follows exactly the same lines as the one provided in [6]. This lemma can be extended without difficulty to the case of a piecewise continuous initial condition $u_{0}$. However, we prefer to restrict our exposition for the sake of simplicity. 
Lemma 3. Provided that $u_{0} \in \mathscr{C}^{n}([-\bar{D}, 0], \mathbb{R})$, the error variable $\varepsilon=u-u^{r}$ with $u$ defined in (14) satisfies the following differential equation for $t \geq 0$

$$
\begin{aligned}
\varepsilon^{(n)}(t)+\left(a_{n-1}\right. & \left.+b_{0} k_{n-1}\right) \varepsilon^{(n-1)}(t)+\ldots+\left(a_{0}+b_{0} k_{0}\right) \varepsilon(t) \\
& =\pi_{1}\left(\dot{D}(t), \ldots, D^{(n)}(t), \varepsilon_{t}, \ldots, \varepsilon_{t}^{(n-1)}, \frac{1}{1+\dot{D}(t)}\right),
\end{aligned}
$$

where $\left[-k_{0} \ldots-k_{n-1}\right] \stackrel{\Delta}{=} K$ and $\pi_{1}$ is a polynomial function which is at least quadratic in the variables $\dot{D}, \ldots, D^{(n)}, \varepsilon_{t}, \ldots, \varepsilon_{t}^{(n-1)}$.

Now that we are equipped with this last lemma, it is possible to use Lemma 2 to guarantee that the stability condition (17) holds.

Lemma 4. Consider the functional $\Theta$ defined in (15). Then, there exists a function $\theta: \mathbb{R}^{n} \mapsto \mathbb{R}_{+}^{*}$ such that, if $\Theta(0)<\theta(K)$, then (17) is fulfilled, which implies that (11) holds.

Proof. The input error $\varepsilon$ satisfies dynamics (28), which is compliant with the assumptions of Lemma 2. In detail, first, the left-hand side of (28) is stable, as it represents the last line of the Hurwitz companion matrix $A+B K$. Second, by observing that $\varphi$ and hence its derivatives are polynomial functions and that

$$
\begin{aligned}
\dot{D}(t)= & \frac{\varphi\left(\varepsilon(t-D(t))+u^{r}\right)-\varphi\left(\varepsilon(t)+u^{r}\right)}{\varphi\left(\varepsilon(t-D(t))+u^{r}\right)} \\
\ddot{D}(t)= & \frac{1}{\varphi\left(\varepsilon(t-D(t))+u^{r}\right)^{3}}\left[\dot{\varepsilon}(t-D(t)) \dot{\varphi}\left(\varepsilon(t-D(t))+u^{r}\right) \varphi\left(\varepsilon(t)+u^{r}\right)\right. \\
& \left.-\dot{\varepsilon}(t) \dot{\varphi}\left(\varepsilon(t)+u^{r}\right) \varphi\left(\varepsilon(t-D(t))+u^{r}\right)\right] \\
D^{(3)}(t)= & \ldots,
\end{aligned}
$$

we obtain by induction that, for $m \geq 1, D^{(m)}$ is a polynomial function in

$$
\varepsilon_{t}, \ldots, \varepsilon_{t}^{(m-1)}, \frac{1}{\varphi\left(\varepsilon(t-D)+u^{r}\right)}
$$

without terms of order 0 or 1 . Therefore, $\pi_{1}$ is directly a polynomial function of the variables

$$
\varepsilon_{t}, \ldots, \varepsilon_{t}^{(n-1)}, \frac{1}{1+\dot{D}}, \frac{1}{\varphi\left(\varepsilon(t-D)+u^{r}\right)},
$$

which is at least quadratic in the variables $\varepsilon_{t}, \ldots, \varepsilon_{t}^{(n-1)}$. Observing that

$$
\frac{1}{1+\dot{D}(t)}=\frac{\varphi\left(\varepsilon(t-D)+u^{r}\right)}{2 \varphi\left(\varepsilon(t-D)+u^{r}\right)-\varphi\left(\varepsilon(t)+u^{r}\right)},
$$

we conclude that this term is continuous and therefore bounded on a given neighborhood of the origin. Hence, as $\pi_{1}$ is at least quadratic, it is possible to properly define a scalar $\omega^{*}>0$ such that

$$
\left|\pi_{1}\left(\varepsilon_{t}, \ldots, \varepsilon_{t}^{(n-1)}\right)\right| \leq c_{*} \max \left|E_{t}\right|, \max \left|E_{t}\right|<\omega^{*},
$$


in which $c_{*}$ is introduced in Lemma 2. This neighborhood depends on the functional $\ell$ and on the constant $c_{*}$ and therefore on the feedback gain $K$. Finally, define

$$
c \ell\left(t, \varepsilon_{t}, \ldots, \varepsilon_{t}^{(n-1)}\right)=\pi_{1}\left(\dot{D}, \ldots, D^{(n)}, \varepsilon_{t}, \ldots, \varepsilon_{t}^{(n-1)}, \frac{1}{1+\dot{D}}\right) .
$$

Then, for $\max \left|E_{t}([-\bar{D}, 0])\right|<\omega^{*}(K)$, we obtain

$$
\left|c \ell\left(t, \varepsilon_{t}, \ldots, \varepsilon_{t}^{(n-1)}\right)\right| \leq c_{*} \max \left|E_{t}\right| .
$$

Therefore, Lemma 2 guarantees the existence of $r_{*}(K)>0$ and $\gamma \geq 0$ such that

$$
\forall t \geq 0, \quad|E(t)| \leq r_{*}(K) \max \left|E_{0}\right| e^{-\gamma t} .
$$

Hence, by choosing

$$
\max \left|E_{0}\right| \leq \frac{1}{r_{*}(K)} \min \left\{\frac{\underline{u} \Delta^{*}(K)}{2 M(\kappa)}, \kappa, \omega^{*}(K)\right\} \triangleq \theta(K)
$$

we ensure that this condition is fulfilled for any $t \geq 0$, that the initial condition lies in the neighborhood $\Omega$ and that

$$
|E(t)| \leq \min \left\{\frac{\underline{u} \Delta^{*}(|K|)}{2 M(\kappa)}, \kappa\right\}, t \geq 0 .
$$

In particular, condition (17) is also fulfilled. Finally, the choice $\max \left|E_{0}\right| \leq \theta(K)$ can be expressed in terms of $\Theta$. This gives the conclusion.

The proof of Theorem 2 directly follows from Lemma 4.

\section{Conclusion}

In this study we have extended some of our recent work to the compensation of input-dependent delay input defined through an integral equation and considered a wide class of kernels (positive polynomial functions of the input). This class of systems is representative of a large number of processes involving transport of material, such as the low-cost rocket design presented above. We prove that robust compensation is achieved by using the current value of the delay as prediction horizon, provided that the initial conditions are in accordance with the magnitude of the feedback gain. The proposed approach is based on a two-step methodology which, first, requires the delay variations to be bounded and, second, relates these variations to input fluctuations. Quantitative comparison of the practical performances of the proposed approach with those of a prediction-based controller using the (constant) final reference delay $D=1 / \varphi\left(u^{r}\right)$ is the natural next step in this approach. Exten- 
sion to explicitly time-dependent delay-integral kernels is also a direction of future work.

\section{References}

1. Artstein, Z.: Linear systems with delayed controls: a reduction. IEEE Transactions on Automatic Control 27(4), 869-879 (1982)

2. Bekiaris-Liberis, N., Krstic, M.: Compensation of state-dependent input delay for nonlinear systems. IEEE Transactions on Automatic Control 58(2), 275- 289 (2013)

3. Bresch-Pietri, D., Chauvin, J., Petit, N.: Adaptive control scheme for uncertain time-delay systems. Automatica 48 (8), 1536-1552, 2012.

4. Bresch-Pietri, D., Chauvin, J., Petit, N.: Invoking Halanay inequality to conclude on closedloop stability of a process with input-varying delay. In: Proceedings of the 10th IFAC Workshop on Time Delay Systems, pp. 266-271 (2012)

5. Bresch-Pietri, D., Chauvin, C., Petit, N.: Prediction-based feedback control of a class of processes with input-varying delay. In: Proceedings of the American Control Conference, pp. 1991-1997 (2012)

6. Bresch-Pietri, D., Chauvin, J., Petit, N.: Sufficient condition for prediction-based stabilization of linear system subject to input-dependent input-delay. In: Proceedings of the American Control Conference, pp. 144-151 (2013)

7. Bresch-Pietri, D., Chauvin, J., Petit, N.: Prediction-based stabilization of linear systems subject to input-dependent input delay of integral-type. IEEE Transactions on Automatic Control 59(9), 2385-2399 (2014)

8. Chebre, M., Creff, Y., Petit, N.: Feedback control and optimization for the production of commercial fuels by blending. Journal of Process Control 20(4), 441-451 (2010)

9. Depcik, C., Assanis, D.: One-dimensional automotive catalyst modeling. Progress in Energy and Combustion Science 31(4), 308-369 (2005)

10. Gu, K., Niculescu, S-I.: Survey on recent results in the stability and control of time-delay systems. Journal of Dynamic Systems, Measurement, and Control 125(2), 158-165 (2003)

11. Halanay, A.: Differential Equations: Stability, Oscillations, Time Lags. Academic Press, New York (1966)

12. Harmand, J., Dochain, D.: The optimal design of two interconnected (bio) chemical reactors revisited. Computers and Chemical Engineering 30(1), 70-82 (2005)

13. Ivanov, A., Liz, E., Trofimchuk, S.: Halanay inequality, yorke $3 / 2$ stability criterion, and differential equations with maxima. Tohoku Mathematical Journal 54(2), 277-295 (2002)

14. Jankovic, M.: Recursive predictor design for linear systems with time delay. In: Proceedings of the American Control Conference, pp. 4904-4909 (2008)

15. Krstic, M.: Boundary Control of PDEs: a Course on Backstepping Designs. Society for Industrial and Applied Mathematics, Philadelphia, PA (2008)

16. Krstic, M.: Delay Compensation for Nonlinear, Adaptive, and PDE Systems. Birkhauser, Boston (2009)

17. Manitius, A., Olbrot, A.: Finite spectrum assignment problem for systems with delays. IEEE Transactions on Automatic Control 24(4), 541-552 (1979)

18. Michiels, W., Niculescu, S-I.: Stability and Stabilization of Time-Delay Systems. Society for Industrial and Applied Mathematics, Philadelphia (2007)

19. Moon, Y., Park, P., Kwon, W.: Robust stabilization of uncertain input-delayed systems using reduction method. Automatica 37(2), 307-312 (2001)

20. Nihtila, M.: Finite pole assignment for systems with time-varying input delays. In: Proceedings of the 30th IEEE Conference on Decision and Control, pp. 927-928 (1991)

21. Perry, R., Green, D., Maloney, J.: Perry's Chemical Engineers' Handbook. McGraw-Hill, New York (1984) 
22. Petit, N., Creff, Y., Rouchon, P.: Motion planning for two classes of nonlinear systems with delays depending on the control. In: Proceedings of the 37th IEEE Conference on Decision and Control, pp. 1007-1011 (1998)

23. Richard, J-P.: Time-delay systems: an overview of some recent advances and open problems. Automatica 39(10), 1667-1694 (2003)

24. Sbarciog, M., De Keyser, R., Cristea, S., De Prada, C.: Nonlinear predictive control of processes with variable time delay. A temperature control case study. In Proceedings of the IEEE International Conference on Control Applications, pp. 1001-1006 (2008)

25. Smith, O.: A controller to overcome dead time. ISA Journal 6(2), 28-33 (1959)

26. Sutton, G., Biblarz, O.: Rocket Propulsion Elements. John Wiley and Sons, New York (2011)

27. Witrant, E.. Stabilisation des Systemes Commandes par Rseaux. PhD Thesis, Laboratoire d'Automatique de Grenoble (2005)

28. Yue, D., Han, Q.: Delayed feedback control of uncertain systems with time-varying input delay. Automatica 41(2), 233-240 (2005) 